\title{
A study on the machining-parameters optimization of wire electrical discharge machining
}

\author{
Y.S. Liao *, J.T. Huang, H.C. Su \\ Department of Mechanical Engineering, National Taiwan University, 1 Sec. 4. Roose'telt Road. Taipei 10764. Taiwan. ROC
}

Received 19 April 1996

\begin{abstract}
A suitable selection of machining parameters for the wire electrical discharge machining (WEDM) process relies heavily on the operators' technologies and experience because of their numerous and diverse range. Machining-parameters tables provided by the machine-tool builder can not meet the operators' requirements, since for an arbitrary desired roughness of the machining surface, they do not provide the optimal machining conditions. An approach to determine parameters setting is proposed. Based on the Taguchi quality design method and the analysis of variance, the significant factors affecting the machining performance such as metal removal rate, gap width, surface roughness, sparking frequency, average gap voltage and normal ratio (ratio of normal sparks to total sparks) are determined. By means of regression analysis, mathematical models relating the machining performance and various machining parameters are established. Based on the mathematical models developed, an objective function under the multi-constraint conditions is formulated. The optimization problem is solved by the feasible direction method, and the optimal machining parameters are obtained. Experimental results demonstrate that the machining models are appropriate and the derived machining parameters satisfy the real requirements in practice. 1997 Elsevier Science S.A.
\end{abstract}

Keywords: Wire electrical discharge machining; Machining-parameters setting; Taguchi quality design: Optimization

\section{Introduction}

Wire electrical discharge machining (WEDM) involves a series of complex physical process including heating and cooling. The electrical discharge energy, affected by the spark plasma intensity and the discharging time, will determine the crater size, which in turn will influence the machining efficiency and surface quality [1-4]. Hence, the machining parameters, including pulse-on time, pulse-off time, table feed rate, flushing pressure, wire tension, wire velocity, etc. should be chosen properly so that a better performance can be obtained. However, the selection of appropriate machining parameters for WEDM is difficult and relies heavily on the operators' experience and machiningparameters tables provided by the machine-tool builder.

Scott, Boyina and Rajurkar [5] used a factorial-design method to determine the optimal combination of control parameters in WEDM, the measures of machin-

\footnotetext{
* Corresponding author. Fax: +886 2 3631755; e-mail: liaoys@cc.ntu.edu.tw
}

ing performance being the metal removal rate and the surface finish. Based on the analysis of variance, it was found that discharge current, pulse duration and pulse frequency are significant control factors for both the metal removal rate and the surface roughness. A total number of 729 experiments was conducted. 32 machining settings which resulted in a better metal removal rate and surface roughness were determined by two distinct techniques: Explicit enumeration of all possible combinations and the dynamic programming approach. However, this approach requires too many experim ${ }^{n}$ ts to be carried out. Further, it can not provide the optimal machining parameters for an arbitrary desired surface roughness or gap width.

Although the effect of improper machining-parameters settings in WEDM could be alleviated by an online control technique, the control reference values, which vary with different machining conditions, are difficult to determine. Moreover, the control effect will be significant only under appropriate off-line machining-parameters setting. The optimal machining-parameters setting based on the operators' experience 
completely does not satisfy the requirements of both high efficiency and good quality. Machining-parameters tables, provided by machine-tool builders. could be a better choice for operators in factory, but still can not be applied to a wide range of machining conditions. Hence, research incorporating physical principles, experimental techniques, mathematical analysis, optimization and computers, is the trend of WEDM optimal machining-parameters setting studies.

Optimal machining-parameters setting for WEDM still has some difficulty from the view of integrity and practice [6,7]. To be more specific, the experiments are too costly and time consuming to meet the requirement of full factorial designs. There are many machining parameters affecting the WEDM machine performance and the real mathematical models between machining performance and machining parameters are not easy to be derived because of the complex machining mechanism. The purpose of this paper is to present an efficient method by means of which to determine appropriate machining parameters so as to be able to achieve the objective of the shortest machining time whilst at the same time satisfying the requirements of accuracy and surface roughness. The cause-effect relationship of machining parameters and machining performance in WEDM is discussed and the significant parameters affecting machining performance are found. Also, the mathematical models relating the machining performance and machining parameters are established by regression and correlation analysis. Finally, the optimal machining parameters by the non-linear approaching method under the constraints and requirements are obtained.

\section{Experimental equipment and design}

A WEDM machine, developed by ITRI (Industrial Technology Research Institute) and CHMER company, Taiwan, was used as the experimental machine. The work material, electrode and the other machining conditions were as follows: (1) workpiece (anode); SKD11 alloy steels; (2) electrode (cathode), $\varnothing 0.25 \mathrm{~mm}$ brass wire; (3) workpiece height, $30 \mathrm{~mm}$; (4) cutting length, $20 \mathrm{~mm}$; (5) open voltage, $95 \mathrm{~V}$; (6) servo reference voltage, $10 \mathrm{~V}$; and (7) specific resistance of fluid, 1-3 mA.

According to the Taguchi quality design concept [8], a L18 mixed orthogonal arrays table was chosen for the experiments. A total of six machining parameters (pulse-on time, pulse-off time, table feed, wire tension, wire speed and flushing pressure) were chosen for the controlling factors and each parameter was designed to have three levels, namely small, medium, and large, denoted by 1, 2 and 3, as shown in Table 1. In order to obtain better mathematical fitting models, another L18 experiments, as given in Table 2 , were conducted.
Table 1

Experimental design I

\begin{tabular}{lcccl}
\hline Control factor & Level 1 & Level & Level 3 & Unit \\
\hline Table feed & 0.4 & 2.5 & & $\mathrm{~mm} \mathrm{~min}{ }^{-1}$ \\
Pulse-on time & 3 & 7 & 10 & $0.1 \mu \mathrm{s}$ \\
Pulse-off time & 6 & 9 & 13 & $1.6 \mu \mathrm{s}$ \\
Wire speed & 6 & 9 & 12 & $\mathrm{~m} \mathrm{~min}$ \\
Wire tension & 800 & 1000 & 1200 & $\mathrm{gf}^{-1}$ \\
Flushing & 3 & 5 & 7 & $\mathrm{~kg} \mathrm{~cm}^{-2 \mathrm{a}}$ \\
\hline
\end{tabular}

a $1 \mathrm{~kg} \mathrm{~cm}^{-2}=98.1 \mathrm{kPa}$.

The machining results after the WEDM process under the designed machining conditions are evaluated in terms of the following measured machining performance: (1) gap width (gap, $\mu \mathrm{m}$ ); (2) metal removal rate (MRR, $\left.\mathrm{mm}^{3} \min ^{-1}\right)$; (3) surface roughness $\left(R_{\mathrm{a}}, \mu \mathrm{m}\right)$; (4) discharging frequency, $\left(F_{\mathrm{q}}, \mathrm{kHz}\right):(5)$ gap voltage $\left(V_{\mathrm{g}}, \mathrm{V}\right) ;(6)$ normal discharge frequency ratio $\left(F_{\mathrm{r}}\right)$.

\section{Experimental results and data analysis}

The chosen six parameters have different influences on the machining performance. The significant parameters are found by the analysis of variance (ANOVA). By regression analysis, mathematical models relating the machining performance to various machining parameters are established and the optimal machiningparameters setting is obtained using the feasible-direction non-linear programming method.

\subsection{The influence of machining parameters on machining performance}

The characteristic that higher value represents better machining performance, such as MRR, is called "higher is better, HB'. Inversely, the characteristic that lower value represents better machining performance, such as surface roughness, is called 'lower is better, LB'. In quality engineering [8], the $S / N$ ratio (signal-to-noise ratio) could be an effective representation to find the significant parameter from those controlling machining

Table 2

Experimental design II

\begin{tabular}{lcrrl}
\hline Control factor & Level 1 & Level 2 & Level 3 & Unit \\
\hline Table feed & 1.2 & 2 & - & $\mathrm{mm} \mathrm{min}-1$ \\
Pulse-on time & 5 & 7 & 8 & $0.1 \mu \mathrm{s}$ \\
Pulse-off time & 8 & 9 & 11 & $1.6 \mu \mathrm{s}$ \\
Wire speed & 8 & 9 & 10 & $\mathrm{~m} \mathrm{~min}-1$ \\
Wire tension & 900 & 1000 & 1100 & $\mathrm{gf}^{-2}$ \\
Flushing & 4 & 5 & 6 & $\mathrm{~kg} \mathrm{~cm}^{-2}$ \\
\hline
\end{tabular}


Table 3

n data for MRR $\left(\mathrm{mm}^{3} \mathrm{~min}\right.$ ',

\begin{tabular}{|c|c|c|c|c|c|c|c|c|c|c|c|c|}
\hline & \multicolumn{8}{|c|}{ Factors } & \multicolumn{3}{|l|}{ MRR } & \multirow{2}{*}{$\frac{n}{\eta(\mathrm{db})}$} \\
\hline & Feed & $T_{(\cdots 1)}$ & $T_{\text {ott }}$ & $v_{n}$ & $F_{w}$ & $P$ & $E_{1}$ & $E_{2}$ & $r_{1}$ & $r=$ & $r_{2}$ & \\
\hline 1 & 1 & 1 & 1 & 1 & 1 & 1 & 1 & 1 & 3.85 & 3.95 & 3.96 & 11.804 \\
\hline 2 & 1 & 1 & 2 & 2 & 2 & 2 & 2 & 2 & 3.84 & 3.83 & 3.9 & 11.723 \\
\hline 3 & 1 & 1 & 3 & 3 & 3 & 3 & 3 & 3 & 3.69 & 3.61 & 3.71 & 11.291 \\
\hline 4 & 1 & 2 & 1 & 1 & 2 & 2 & 3 & 3 & 5.12 & 5.03 & 5.1 & 14.122 \\
\hline 5 & 1 & 2 & 2 & 2 & 3 & 3 & 1 & 1 & 4.71 & 4.66 & 4.64 & 13.386 \\
\hline 6 & 1 & 2 & 3 & 3 & 1 & 1 & 2 & 2 & 5.16 & 5.05 & 5.24 & 14.233 \\
\hline 7 & 1 & $?$ & 1 & 2 & 1 & 3 & 2 & 3 & 5.7 & 5.34 & 5.49 & 14.814 \\
\hline 8 & 1 & 3 & 2 & 3 & 2 & 1 & 3 & 1 & 5.28 & 5.28 & 5.4 & 14.517 \\
\hline 9 & 1 & 3 & 3 & 1 & 3 & 2 & 1 & 2 & 6.36 & 7.28 & 6.55 & $\mathbf{i} 6.517$ \\
\hline 10 & 2 & 1 & 1 & 3 & 3 & 2 & 2 & 1 & 4.75 & 4.61 & 4.55 & 13.32 \\
\hline 11 & 2 & 1 & 2 & 1 & 1 & 3 & 3 & 2 & 5.35 & 5.72 & 5.47 & 14.818 \\
\hline 12 & 2 & 1 & 3 & 2 & 2 & 1 & 1 & 3 & 4.96 & 5.17 & 4.91 & 13966 \\
\hline 13 & 2 & 2 & 1 & 2 & 3 & 1 & 3 & 2 & 31.02 & 31.77 & 31.84 & 29.976 \\
\hline 14 & 2 & 2 & 2 & 3 & 1 & 2 & 1 & 3 & 30.97 & 31.03 & 31.99 & 29.916 \\
\hline 15 & 2 & 2 & 3 & 1 & 2 & 3 & 2 & 1 & 32.35 & 32.39 & 32.24 & 30.191 \\
\hline 16 & \pm & 3 & 1 & 3 & 2 & 3 & 1 & 2 & 33.92 & 34.36 & $3+.59$ & 30.702 \\
\hline 17 & 2 & 3 & 2 & 1 & 3 & 1 & 2 & 3 & 28.32 & 28.98 & 28.94 & 29.17 \\
\hline 18 & 2 & 3 & 3 & 2 & 1 & 2 & 3 & 1 & 30.74 & 30.01 & 30.66 & 29.676 \\
\hline
\end{tabular}

parameters by evaluating tie minimum variance. For $H B$ and LB, dae defintion or the $S N$ ration for machining-performanec results $y$, of $n$ repoated number on this case $n=3, i=1,2,3)$ are

HB: $\quad S / N$ ratio $=1 \sigma^{2} . \quad \sigma^{2}=\frac{1}{n}\left(\frac{1}{y}+\frac{1}{y}+\cdots+\frac{1}{y}\right)$

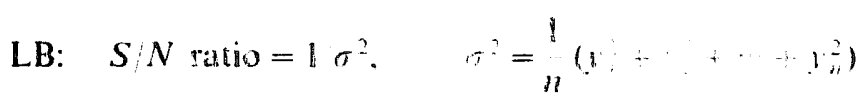

In Taguchi quality engineering [8], the $\eta$ walue is a better representation than the $S / N$ ratio. Larger $\eta$ (or a larger $S / N$ ratio equivalently) is better for both $\mathrm{HB}$ and LB.

$\eta=10 * \log (S / N$ ratio $)$

By applying the equation above, the $\eta$ values of machining performance for each experiment of L18 can be calculated. Taking the metal removal rate (MRR) as an example, the $\eta$ values are computed and given in Table 3.

In order to obtain the effects of machining parameters on machining performance for each different level, the $\eta$ values of each fixed parameter and level for each machining performance are summed up. From Table 3 , taking $T_{\text {on }}$ on MRR as an example, the $\eta$ values of three levels can be summarized as follows:

Level 1:

$$
\begin{aligned}
\eta_{\mathrm{on} 1}= & 11.864+11.723+11.291+13.320+14.818 \\
& +13.996=77.012
\end{aligned}
$$

Level 2:

$$
\begin{aligned}
\eta_{10122}= & 14.122+13.386+14.233+29.976+29.916 \\
& +30.191=131.824
\end{aligned}
$$

Level 3:

$$
\begin{aligned}
\eta_{1 \ldots 1:}= & 14.814+14.517+16.517+30.702+29.170 \\
& +29.676=135.396
\end{aligned}
$$

Similarly, those $\eta$ values of the other parameters on other machining performance could be evaluated. Table 4 shows the total $\eta$ values of the levels of six parameters on MRR.

\subsection{Analysis of variance}

From Table 4, the combination of machining parameters (Feed) ${ }_{2} T_{\mathrm{om} 3} T_{\mathrm{om} 3} V_{\mathrm{wi}} F_{\mathrm{w1}} P_{2}$ would result in maximum MRR because of larger $\eta$ values. However, it is hard to assure that only this setting will result in maximum MRR because of the small difference of the " value between different levels and experimental error. Hence, analysis of variance (ANOVA) and the $F$ test are used to analyze the experimental data as follows:

$S_{\mathrm{m}}=\frac{\left(\sum \mu_{i}\right)^{2}}{18}, \quad S_{\mathrm{T}}=\sum \eta_{i}^{2}-S_{\mathrm{m}}$

$S_{A}=\frac{\sum \eta_{A i}^{2}}{N}-S_{\mathrm{m}}, \quad S_{\mathrm{E}}=S_{\mathrm{T}}-\sum S_{A t}$ 
Table 4

Totil $\eta$ values of each level on MRR

\begin{tabular}{lllllll}
\hline Level & Feed & $T_{\text {on }}$ & $T_{\text {off }}$ & $V_{w}$ & $F_{w}$ & $P$ \\
\hline 1 & 122.467 & 77.012 & 114.798 & 116.682 & 115.321 & 113.756 \\
2 & 221.765 & 131.824 & 113.53 & 113.571 & 115.251 & 115.274 \\
3 & & 135.396 & 115.904 & 113.979 & 113.66 & 115.202 \\
Sum & 344.232 & 344.232 & 344.232 & 344.232 & 344.232 & 344.232 \\
\hline
\end{tabular}

$V_{A}=\frac{S_{A}}{f_{A}}, \quad F_{A 0}=\frac{V_{A}}{V_{\mathrm{E}}}$

where $S_{\mathrm{T}}$ is the sum of squares due to total variation; $S_{\mathrm{m}}$ is the sum of squares due to mean; $S_{A}$ is the sum of squares due to factor $A$ ( $A=$ Feed, $T_{\mathrm{on}}, T_{\mathrm{off}}, V_{\mathrm{w}}, F_{\mathrm{w}}$ and $P$ ); $S_{\mathrm{E}}$ is the sum of squares due to error; $\eta_{i}$ is the $\eta$ value of each experiment $(i=1-18) ; \eta_{A i}$ is the sum of $i$ level of factor $A(i=1,2$ or $i=1,2,3) ; N$ is the repeating number of each level of factor $A ; f_{A}$ is the degree of freedom of factor $A ; V_{A}$ is the variance of factor $A$; and $F_{A 0}$ is the $F$ test value of factor $A$.

The square sum, variance and $F$ test value of machining parameters on the MRR in Table 5 were obtained hy substituting those data in Tables 3 and 4 into the above equations. The bold type in Table 5 indicates the significant factors on MRR being Feed and $T_{\text {on }}$ for the reason that both of the $F_{A 0}$ values are bigger than $F_{0.05, m 1, n 2}$. Similarly, the significant parameters for each machining performance are shown in Table 6 .

In order that the effects of machining parameters on machining performance can be seen more clearly, the data in Table 4 were plotted as shown in Figs. 1-3. It is found from Fig. 1 that larger Feed and $T_{\text {on }}$ results in larger MRR (HB) and from Fig. 2 larger $T_{\text {on }}$ results in larger gap width (LB). For surface roughness (LB), larger $T_{\mathrm{on}}$ result in a bigger crater on the surface (Fig. 3). No significant parameter was found on normal discharge frequency ratio $\left(Y_{\mathrm{r}}\right)$, one of the reasons being that all parameters have little effect on $Y_{r}$, whilst another more likely reason is that the effects of the error factor on the experimental results exceed those of all of these machining parameters. The trends of the influence of significant parameters on machining performance are summarized in Table 7.

\subsection{Mathematical model of machining performance}

By regression and correlation analysis, the mathematical models are obtained as follows:

$$
\begin{aligned}
Y_{\mathrm{mr}}= & 18.5953 x_{1}^{2} x_{2}-3.5215 x_{1}^{2} x_{2}^{2}-17.7297 x_{1} x_{2} \\
& -5.4441 x_{1}+5.7634 x_{2}+3.2558 \\
Y_{\text {gap }}= & 0.5097 x_{2}+0.4406 \\
Y_{\mathrm{SR}}= & 0.6891 x_{2}^{2}-0.2698 x_{2}+0.5725
\end{aligned}
$$

$$
\begin{aligned}
Y_{\mathrm{fq}}= & -0.0148 x_{1} x_{2}^{2}+9.4631 x_{1}^{2} x_{2}-18.3852 x_{1} x_{2} \\
& +3.0855 x_{1}+7.8901 x_{2}-1.7051 \\
Y_{\mathrm{vg}}= & -6.3851 x_{2}^{2}+7.1263 x_{1} x_{2}^{2}-10.5043 x_{1}^{2} x_{2} \\
& +10.5767 x_{1} x_{2}-0.0287
\end{aligned}
$$

where $x_{1}$ and $x_{2}$ are calculated by the following equations:

$x_{1}=\log ($ Feed $) / \max (\log ($ Feed $))$

$x_{2}=\log \left(T_{\mathrm{on}}\right) / \max \left(\log \left(T_{\mathrm{on}}\right)\right)$

Because:

$400 \leq$ Feed $\leq 2500, \quad 3 \leq T_{\text {on }} \leq 10$

then:

$0.7657 \leq x_{1} \leq 1, \quad 0.4771 \leq x_{2} \leq 1$

Evidence shows that the mathematical models derived by the regression analysis above are sufficiently precise to represent the real machining performance. Presented are the correlation coefficients of experimental data $\left(Y_{i}\right)$ and data $\left(Y_{i}^{\prime}\right)$ calculated by the regression mathematical model.

$\operatorname{coef}\left(Y_{\mathrm{mr}}, Y_{\mathrm{mr}}^{\prime}\right)=0.9883$

$\operatorname{coef}\left(Y_{\text {gap }}, Y_{\text {gap }}^{\prime}\right)=0.9054$

$\operatorname{coef}\left(Y_{s r}, Y_{s r}^{\prime}\right)=0.9652$

$\operatorname{coef}\left(Y_{\mathrm{fq}}, Y_{\mathrm{fq}}^{\prime}\right)=0.9598$

$\operatorname{coef}\left(Y_{\mathrm{vg}}, Y_{\mathrm{vg}}^{\prime}\right)=0.9601$

Another correlation-coefficient analysis between various machining performances was evaluated as given in Table 8, which table shows that a larger MRR will be accompanied by a bigger $R_{\mathrm{a}}$. A larger gap width will accompany a bigger $R_{\mathrm{a}}$ and gap voltage and the total discharge frequency will increase whilst the gap voltage decreases. It is impossible to achieve a higher MRR and good surface phenomena simultaneously. Therefore, the optimum machining parameters setting will be presented in the next section.

\subsection{The optimization of machining parameters settings}

The feasible-direction method of non-linear programming was applied to find the optimum machining set- 
Table 5

ANOVA for MRR

\begin{tabular}{|c|c|c|c|c|c|}
\hline Factor $(A)$ & $\operatorname{Degrec}\left(f_{1}\right)$ & Square sum $\left(S_{A}\right)$ & Variance $\left(l l_{1}\right)$ & $F .10$ & $F_{11, \ldots, n, \ldots 2}$ \\
\hline Feed & 1 & 547.7829 & 547.7829 & 19.9955 & 5.99 \\
\hline$T_{\text {on }}$ & 2 & 356.9892 & 178.4946 & 6.2223 & 5.14 \\
\hline$T_{o t:}$ & 2 & 0.4704 & 0.2352 & 0.0082 & 5.14 \\
\hline$V_{w}$ & 2 & 0.9528 & 0.476 & 0.0166 & 5.14 \\
\hline$F_{w}$ & 2 & 0.2941 & 0.1471 & 0.0051 & 5.14 \\
\hline$P$ & 2 & 0.2444 & 0.1222 & 0.0043 & 5.14 \\
\hline$E_{\mathrm{u} 1+02}$ & 6 & 172.1185 & 28.6864 & & \\
\hline$T$ & 17 & 1078.852 & & & \\
\hline
\end{tabular}

Bold type indicates significant factors.

ting by seeking the maximum value of the objective function under some constraints [9]. A programmed software was adopted to solve above problem.

Referring to Fig. 4, first an initial set of design variable $X^{0}$ is required to be specified as the starting point in the design space. Beginning from this starting point, the variable vector is updated iteratively by using the iterative form given by:

$X^{q}=X^{q-1}+x^{*} \cdot S^{q}$

where $X^{\prime \prime}$ is the optimal variable solution and $S^{\prime \prime}$ is a usable-feasible search direction vector in the design space in no. $q$ iteration. The scalar quantity $x^{*}$ defines the distance that is desired to be moved in the direction $S$. The task is to find a search direction $S$ which will reduce the obiective function without violating the active constraint for some finite move. Any $S$ vector which reduces the objective function is called the usable direction. Therefore, if the objective function is denoted by $F(X)$, then the dot product of the gradient of $F\left(X^{\prime \prime}\right)$. $\nabla F\left(X^{\prime \prime}\right)$ and $S$ should be negative because the angle between them exceeds $90^{\circ}$. Mathematically, the usability requirement becomes:

$\nabla F\left(X^{0}\right) \cdot S \leq 0$

and a direction is called feasible if, for some small move in that direction, the active constraint will not be violated, i.e. the dot product of the gradient of $g_{1}\left(X^{0}\right)$, $\nabla g_{1}\left(X^{0}\right)$ and $S$ must be non-positive. Here $g_{1}(X)$ is the constraint function. Thus:

Table 6

The influence of machining parameters on machining performance

\begin{tabular}{|c|c|c|c|c|c|c|}
\hline & $Y_{\mathrm{mir}}$ & $Y_{\text {gar }}$ & $Y_{\mathrm{sr}}$ & $Y_{\mathrm{ti}_{4}}$ & $Y_{1:}$ & $Y_{\mathrm{r}}$ \\
\hline Feed & $\downarrow$ & & & 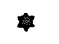 & 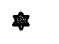 & \\
\hline$T_{\mathrm{on}}$ & $\star$ & $\star$ & * & $\#$ & $\not$ & $\dot{t}$ \\
\hline$T_{\text {eff }}$ & & & 4 & $\therefore$ & $t$ & \\
\hline $\begin{array}{l}V_{\mathrm{w}} \\
F_{\mathrm{w}} \\
P\end{array}$ & $\hat{s}$ & $\hat{s}$ & & & & \\
\hline
\end{tabular}

\$, Significant parameter: 虫, sub-significant parameter. $\nabla g_{1}\left(X^{\prime \prime}\right) \cdot S \leq 0$

In this paper, the feasible-direction non-linear programming method is applied to show how the optimal machining parameters setting which will result in the maximum metal removal rate under constraints (surface roughness and gap limitations for examples) can be obtained.

For illustration purposes, if it is required to maximize the metal removal rate under the constraints of a gap width smaller or equal to $0.42 \mathrm{~mm}$, and the surface roughness should be less or equal to $3.0 \mu \mathrm{m}$, then mathematically, this problem is expressed as:

Maximize: $\quad Y_{\mathrm{mr}}$

Subject to:

$$
\begin{aligned}
& Y_{\text {gut }} \leq 0.42 \mathrm{~mm} \\
& Y_{\mathrm{sr}} \leq 3.0 \mu \mathrm{m} \\
& \left(x_{1}-1\right)\left(x_{1}-0.7675\right) \leq 0 \\
& \left(x_{2}-1\right)\left(x_{2}-0.4771\right) \leq 0
\end{aligned}
$$

The non-linear optimization yields:

Feed $=2.48\left(\mathrm{~mm} \mathrm{~min}^{-1}\right) \quad T_{\text {on }}=8.13(0.1 \mu \mathrm{s})$

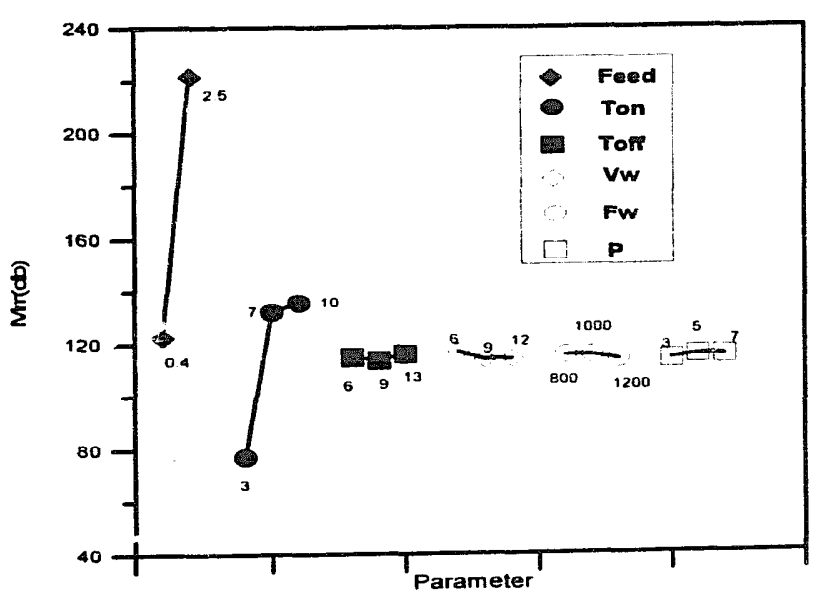

Fig. 1. The influence of machining parameters on the MRR. 


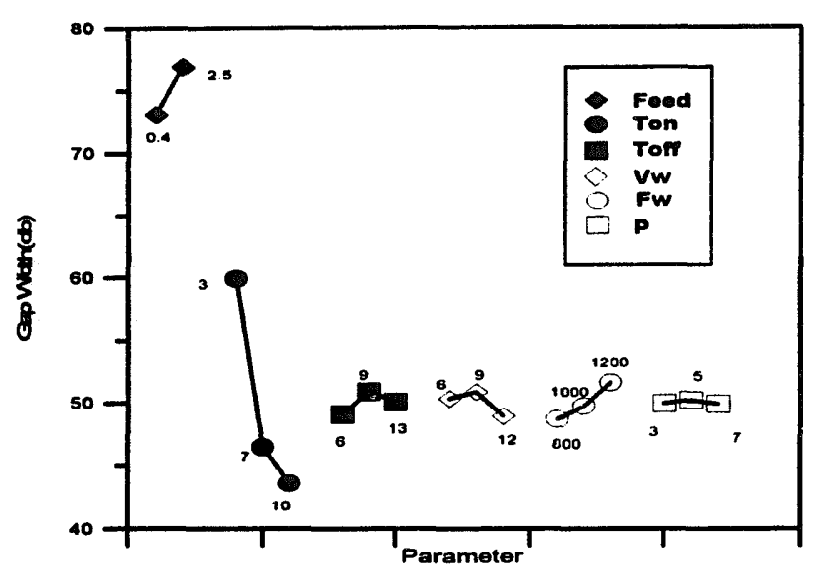

Fig. 2. The influence of machining parameters on the gap width.

In practice, if the following are chosen: Feed $=2.5(\mathrm{~mm}$ $\left.\min ^{-1}\right), T_{\text {on }}=8(0.1 \mu \mathrm{s}), T_{\text {off }}=9(1.6 \mu \mathrm{s}), V_{\mathrm{w}}=9 \mathrm{~m}$ $\min ^{-1}, F_{\mathrm{w}}=1000 \mathrm{gf}, P=5 \mathrm{~kg} \mathrm{~cm}^{-2}$; then the actual experiments result in:

$Y_{\mathrm{mr}}=34.577\left(\mathrm{~mm}^{3} \mathrm{~min}^{-1}\right) \quad Y_{\text {gap }}=0.403(\mathrm{~mm})$

$Y_{\mathrm{sr}}=2.94(\mu \mathrm{m}) \quad Y_{\mathrm{f} \mathrm{Y}}=25.833(\mathrm{kHz})$

$Y_{\mathrm{vg}}=61.235(\mathrm{~V})$

The metal removal rate is maximized whilst the gap width and surface roughness constraints are satisfied for a table feed of between 0.4 and $2.5 \mathrm{~mm} \mathrm{~min}^{-1}$ and $T_{\text {on }}$ between 3 and $10(0.1 \mu \mathrm{s})$.

\section{Conclusions}

A methodology to determine the optimal machining parameters setting in WEDM was proposed. This methodology is not only time saving and cost effective but also efficient and precise in determining the machining parameters.

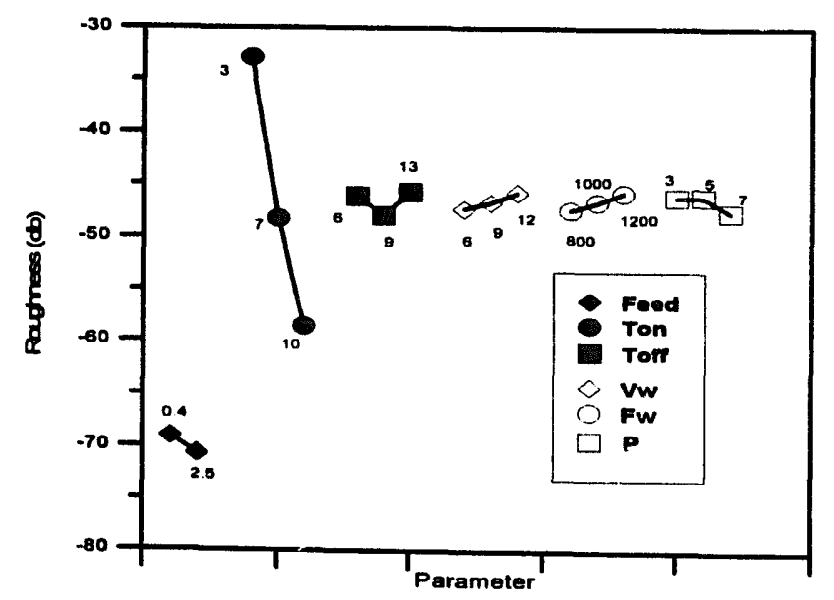

Fig. 3. The influence of machining parameters on the surface roughness.
Table 7

The trends of the influence of parameters on machining performance

\begin{tabular}{lllllll} 
& $Y_{\text {ir }}$ & $Y_{\text {zap }}$ & $Y_{\mathrm{sr}}$ & $Y_{\mathrm{lu}}$ & $Y_{\mathrm{v}}$ & $Y_{\mathrm{r}}$ \\
\hline Feed & $\nearrow$ & & & $\nearrow$ & $\searrow$ \\
$T_{\text {on }}$ & $\nearrow$ & $\nearrow$ & $\nearrow$ & $\searrow$ & $\nearrow$
\end{tabular}

$\nearrow$. Performance increasing as increasing Feed or $T_{o n}$.

. Performance decreasing as increasing Feed or $T_{o n}$.

Table 8

Correlation coefficients between machining performance

\begin{tabular}{|c|c|c|c|c|c|}
\hline & $Y_{\mathrm{mr}}$ & $Y_{\mathrm{z}: \mathrm{p}}$ & $Y_{\mathrm{sr}}$ & $Y_{\mathrm{t}_{4}}$ & $Y_{\mathrm{g}}$ \\
\hline$Y_{\mathrm{aur}}$ & 1 & & & & \\
\hline$Y_{\text {e:ap }}$ & 0.2429 & 1 & & & \\
\hline$Y_{\mathrm{sr}}$ & 0.4689 & $0.870 !$ & 1 & & \\
\hline$Y_{\mathrm{fy}}$ & 0.1594 & -0.7945 & -0.5898 & 1 & \\
\hline$Y_{\text {sy }}$ & -0.0754 & 0.8178 & 0.636 & -0.9496 & 1 \\
\hline
\end{tabular}

It is found the table feed and pulse-on time have a significan! influence on the metal removal rate, the gap voltage and the total discharge frequency, whilst the gap width and the surface roughness are mainly influenced by the pulse-on time. Therefore, adjusting the table feed and $T_{\text {on }}$ is an appropriate strategy to control the discharging frequency for the prevention of wire breakage. A larger table feed and a smaller $T_{\text {on }}$ are recommended for the reason that a longer $T_{\text {on }}$ will result in a higher value of $R_{\mathrm{a}}$. However, this does not take place for a larger Feed, although the table feed cannot be increased without constraints because of the risk of wire breakage.

In the future, the methodology presented in this paper could be applied to different machining condition such as different work material, electrode, etc. so as to build a CAPP expert system of WEDM with the goal of automation.

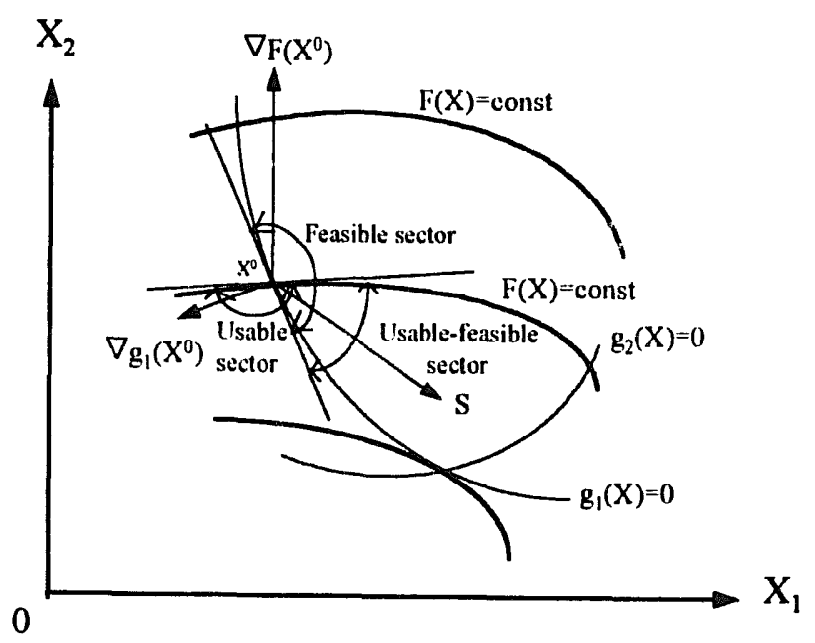

Fig. 4. Usable-feasible search direction [9]. 


\section{Nomenclature}

Feed table feed

$T_{\text {on }} \quad$ pulse-on time

$T_{\text {,ifl }} \quad$ pulse-off time

$V_{\mathrm{u}} \quad$ wire speed

$F_{\mathrm{w}} \quad$ wire tension

$P \quad$ flushing pressure

$Y_{\text {gap }} \quad$ gap width

$Y_{\mathrm{mr}} \quad$ metal removal rate

$Y_{\mathrm{sr}} \quad$ surface roughness

$Y_{\mathrm{fu}} \quad$ discharge frequency

$Y_{\mathrm{vg}} \quad$ gap voltage

$Y_{\mathrm{r}} \quad$ normal discharge frequency ratio

\section{References}

[1] G.N. Levy, F. Maggi, WEDM machinability comparison of different steel grades, Ann. CIRP 39 (1) (1990) 183185.
[2] 1. Ogata. Y. Mokoyama. Residual stress on surfite machined by wire electric discharge, Int. J. Japan Soc. Prec. Eng. $25(4) 11991$ ) $273-278$.

[3] R.E. Williams, K.P. Rajurkar. Study of wire electrical discharge machined surface characteristics. J. Mater. Proc. Technol. 28 (1991) $127-138$.

[4] Y. Suziki. M. Kishi. Improvement of surface roughness in wire EDM, in: Proceedings of the International Symposium for Electro-Machining, (ISEM-9) Nagoya, 1989, pp. 80-83.

[5] D. Scott, S. Boyina, K.P. Rajurkar, Analysis and optimization of parameter combination in wire electrical discharge machining, Int. J. Prod. Res. 29 (11) (1991) 2189-2207.

[6] M.S. Phadke, Quality Engineering Using Robust Design. Prentice Hall, Englewood Cliffs, NJ, 1989.

[7] J. Matsuda, S. Tomishige, K. Tanaka, H. Yano. Evaluation of performance of WEDM by parameter design, in: Proceedings of the International Symposium for Electro-Machining. (ISEM-9) Nagoya, 1989, pp. 68 71 .

[8] P.J. Ross, Taguchi Techniques for Quality Engineering. McGraw-Hill, New York, 1989.

[9] G.N. Vanderplats. Numerical Optimization Techniques for Engineering Design: With Applications. McGraw-Hill. New York. 1984. 